\title{
Miniinvasive hybrid procedure for device migration after percutaneous closure of persistent arterial duct: a case report
}

\author{
Ireneusz Haponiuk ${ }^{1}$, Maciej Chojnicki', Radoslaw Jaworski ${ }^{1}$, Mariusz Steffek ${ }^{1}$, Jacek Juscinski ${ }^{1}$, Irena Zabolska ${ }^{1}$, \\ Aneta Sendrowska ${ }^{1}$, Katarzyna Gierat-Haponiuk ${ }^{2}$ \\ 1Department of Paediatric Cardiac Surgery, Mikołaj Kopernik Pomeranian Centre of Traumatology, Gdansk, Poland \\ 2Department of Rehabilitation, Medical University of Gdansk, Poland
}

\begin{abstract}
We report a case of an 8-month-old girl admitted to the Department of Paediatric Cardiac Surgery, Pomeranian Centre of Traumatology in Gdansk with migration of an Amplatzer Duct Occluder II device (AGA Med. Corp., USA) to the left pulmonary artery after interventional patent arterial duct (PDA) closure. Using a hybrid strategy, we performed a classical surgical closure of the PDA with simultaneous intraoperative miniinvasive catheter removal of the displaced implant from the left pulmonary artery using a muscle bioptome (Cook, EU). The procedure was successful, without any further complications. Percutaneous procedures of PDA closure in small children, although safe and effective, are associated with a risk of accompanying complications, especially in patients with inconvenient anatomy. Our strategy demonstrates that a miniinvasive hybrid strategy could be beneficial for the patient with implant PDA migration after a failed interventional procedure. We strictly maintain the practice of qualifying these borderline patients with great care to avoid predictable complications, and to provide immediate surgical support in any emergency, following modern models of cooperation between cardiologists and cardiac surgeons in hybrid heart teams. A modern strategy that combines miniinvasive cardiac surgery with interventional techniques provides new, effective algorithms for selective difficult clinical settings.
\end{abstract}

Key words: patent arterial duct, device closure, miniinvasive hybrid procedures, congenital heart defects, cardiac surgery.

\section{Introduction}

Transcatheter closure of persistent arterial duct (PDA) is a well-established minimally invasive technique that has become a real alternative to surgery, especially in children over 1 year old [1] The success of detachable coils in the closure of small diameter ducts turned interest towards embolization of larger communications, with great progress after the introduction of Amplatzer devices [2]. The Amplatzer Duct Occluders II (ADO) (AGA Med. Corp., USA) were implanted with good results including in children under 6 months and body mass even less than $6 \mathrm{~kg}$. The main advantages of ADO were small diameters of the detachment system and lower incidence of residual leaks, embolization, haemolysis, and left pulmonary artery (LPA) stenosis [3]. The use of ADO, although safe and effective in the closure of arterial ducts in small babies, remains a difficult procedure in inconvenient anatomy and large diameters of the PDA. In this group of selected patients the procedure of choice still remains surgical ligation, while vain attempts to implant the device in borderline indications could result in troublesome complications [4]. 
We present a case of complete left pulmonary embolization as a consequence of implant migration in a child referred for percutaneous PDA closure in a different institution, who was sent for emergency surgery to the Department of Paediatric Cardiac Surgery in Gdansk, with concomitant symptoms of left lung hypoperfusion as well as local necrosis in the lower limb after femoral vessel cannulations.

\section{Case report}

The 8-month-old girl (body weight $5.2 \mathrm{~kg}$ ) was admitted to the Department of Paediatric Cardiac Surgery, Pomeranian Centre of Traumatology in Gdansk, with the diagnosis of a large $4 \mathrm{~mm}$ PDA and left-to-right shunt after failed percutaneous closure, with the ADO II (AGA Med. Corp., USA) implant migrated to the main pulmonary artery (PA). There was also deep skin necrosis of the right shin, probably as the result of right femoral vessel cannulation with the ADO release system. Following the initial diagnosis we were expecting the device in the main pulmonary artery, but precise echocardiography and preoperative chest X-ray (Figure 1) showed the displaced ADO device occluding the left pulmonary artery (LPA) in the left hilar space, at a distance of $8 \mathrm{~mm}$ away from the PA bifurcation. The shunt via the PDA was still significant with high PA pressure and mild pulmonary valve insufficiency (PVI), and a significant reduction of the LPA flow with left pulmonary vein return was noted. The girl was qualified for a surgical procedure of PDA closure with simultaneous miniinvasive removal of the implant via midline sternotomy, to provide an additional safeguard with extracorporeal circulation (ECC) on-side in case of any unexpected complications. The procedure was done immediately after the preoperative heparin administration $(1.5 \mathrm{mg} / \mathrm{kg})$ to reduce the risk of pulmonary thromboembolization. After a classic midline sternotomy and purse-strings preparation for ECC cannulation the large-diameter PDA was doubly ligated with an additional vascular clip. Afterwards the caval snares were tightened (following the inflow occlusion technique) and the implant was harvested from the left pulmonary artery using a muscle bioptome (Cook, EU). The harvesting wire was inserted into the main PA through a separate purse-string suture, and directed towards the LPA bifurcation under fluoroscopic control. After the manoeuvres the haemostasis was done without heparin reversion to avoid any risk of postoperative thrombus.
The early postoperative course was standard and uneventful. The heparin infusion was continued under activated partial thromboplastin time (APTT) control followed by oral Aspirin administration for the next 7 days. Control echocardiography proved complete PDA closure with normal left lung perfusion and heart function recovery. The deep skin necrosis in the right shin was treated as a separate procedure with autologous skin transplantation staged therapy up to 6 months after the cardiac procedure in the paediatric surgery department.

\section{Discussion}

The first successful reports of interventional Amplatzer device PDA closure in children were reported in 1998 [2]. After that, interventional PDA closure using the ADO became a commonly used method of choice, even in infants, with the great advantages of small diameter of the release systems and conical shape of the device that facilitate its use in large ducts [4]. However, all interventional procedures are still not free of technical complications and related disadvantages. The most common complications of ADO are device embolization, left pulmonary stenosis, implant migration, and even intraoperative

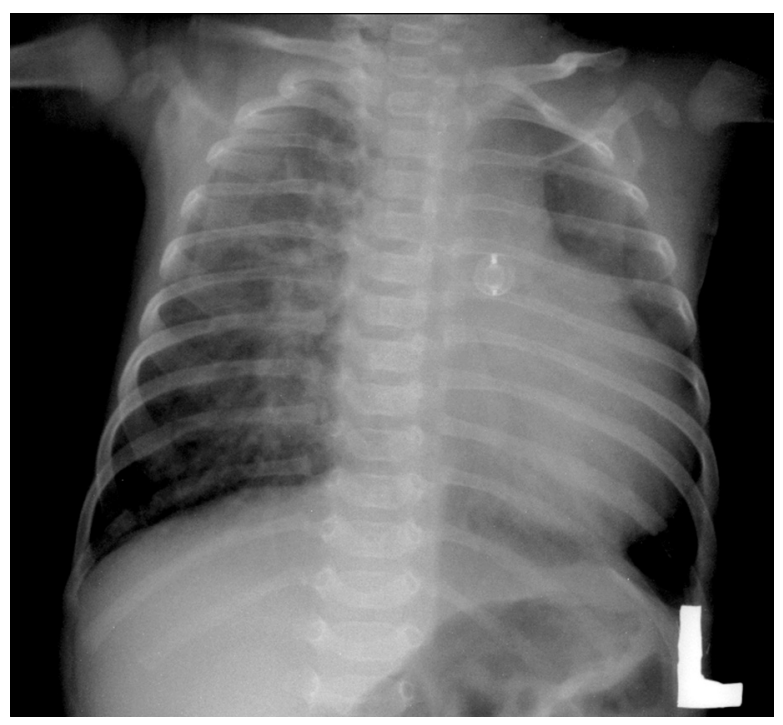

Figure 1. Preoperative X-ray image with migrated Amplatzer duct occluder shape in the left pulmonary artery area. Note that there is marked disproportion between excessive pulmonary bed on the right and the lack of vasculature seen in the left lung 
deaths. Other complications are transient loss of arterial pulse after procedure, blood loss requiring transfusion, haematoma, complete heart block, ventricular tachyarrhythmia, hypotension, injury of the aortic valve, aortic obstruction, cardiac arrest, cardiac perforation, stroke and device-related haemolysis $[3,4]$. There are also reports of embolization caused by device displacement to the pulmonary artery. In such a case, the device migrates mostly to the right pulmonary artery or stops in the pulmonary bifurcation [5]. In the reviewed literature we did not find any reports of device displacement into the left pulmonary artery, like in the presented case. In such circumstances an attractive therapeutic option is a miniinvasive surgical hybrid procedure, that enables simultaneous PDA ligation as well as safe removal of the migrated device using a muscle bioptome through the well-exposed main pulmonary artery. In order to achieve maximum safety for the patient an additional safeguard with emergency ECC on-side in case of any complications was prepared.

Surgical backup is still necessary for safe interventional treatment, while in some teams conventional surgical ligation remains the procedure of choice [6]. Following the literature reports as well as individual experience, hybrid therapies, which combine the advantages of surgical and interventional techniques, are especially beneficial in borderline patients, who could not complete selective qualification criteria for either surgery or cardiac interventions [7]. In the presented patient a miniinvasive hybrid approach had the advantage of avoiding ECC and haemodynamic instability in a small, complicated baby with left lung hypoperfusion. All steps of the procedure had to be accomplished gently, with minimal manipulation and invasiveness, to prevent any clot mobilization and further peripheral pulmonary embolization.

Although the ADO implantation has become a procedure of choice in small babies, it is twice as important to mention the great role of the correct preoperative qualification, especially in small individuals with large, haemodynamically important leftto-right shunts through the PDA with regard to Krichenko's classification [8]. Possible mismatch between implanted occluder size and anatomical PDA diameters could be the reason for the phenomenon of PDA underestimation and subsequent complications. The question still needs to be answered which strategy would be the best option for small children: an attractive miniinvasive PDA interventional closure or a classic surgical approach, both options with their advantages as well as predictable complications [4, 6]. Peripheral hypoperfusion symptoms prove the mismatch between small diameter of the groin vessels, which were probably occluded by the introduction system of the device, with necrotic complications requiring surgery.

In our material the Amplatzer Duct Occluder II is the device of choice for percutaneous PDA closure in small babies. We also reported an alternative use of ADO II for intraoperative hybrid muscular VSD (mVSD) closure in the case of a premature baby aged 2 months (body weight $3.4 \mathrm{~kg}$ ) because of its small discs, which did not interfere with intracardiac structures in the small heart [7]. Exactly for these reasons the ADO II was used for percutaneous obliqueshaped residual mVSD closure in a child after surgery for large perimembranous VSD closure, although we routinely implant the Amplatzer VSD Occluder (AGA Med. Corp., USA) [9].

In conclusion, our strategy demonstrates that miniinvasive hybrid strategy could be beneficial for the patient with implant PDA migration after a failed interventional procedure. A modern strategy that combines miniinvasive cardiac surgery with interventional techniques provides new effective algorithms for selective difficult clinical settings, on condition that the crucial cooperation between the cardiac surgeon and the interventional cardiologist is excellent.

\section{Acknowledgments}

We gratefully acknowledge the support of Dr Mark Galantowicz and Dr John Cheatham from the Nationwide Children's Hospital in Columbus, Ohio, USA, who made available fundamental skills and spirit for the formation of the Gdansk Hybrid Heartlink Programme in the Department of Paediatric Cardiac Surgery, Pomeranian Centre of Traumatology in Gdansk, Poland.

\section{References}

1. Kusa J, Szkutnik M, Baranowski J, et al. Percutaneous closure of recanalised ductus arteriosus: a single-centre experience. Kardiol Pol 2007; 65: 125-9.

2. Masura J, Walsh KP, Thanopoulous B, et al. Catheter closure of moderate- to large-sized patent ductus arteriosus using the new Amplatzer duct occluder: immediate and short-term results. J Am Coll Cardiol 1998; 31: 878-82. 
3. Parra-Bravo R, Cruz-Ramirez A, Rebolledo-Pineda V, et al. Transcatheter closure of patent ductus arteriosus using the amplatzer duct occluder in infants under 1 year of age. Rev Esp Cardiol 2009; 62: 867-74.

4. Abadir S, Boudjemline Y, Rey C, et al. Significant persistent duCtus arteriosus in infants less or equal to $6 \mathrm{~kg}$ : percutaneous closure or surgery? Arch Cardiovasc Dis 2009; 102: 533-40.

5. Amanullah MM, Siddiqui MT, Khan MZ, Atig MA. Surgical rescue of embolized amplatzer devices. J Card Surg 2011; 26: 254-8.

6. Ewert P. Challenges encountered during closure of patent ductus arteriosus. Pediatr Cardiol 2005; 26: 224-9.

7. Haponiuk I, Chojnicki M, Jaworski R, et al. Miniinvasive hybrid closure of multiple muscular ventricular septal defects in premature infant with a novel use of Amplatzer Duct Occluder II a case report. Videosurgery Miniinv 2011; 6: 33-6.

8. Krichenko A, Benson LN, Burrows P, et al. Angiographic classification of the isolated, persistently patent ductus arteriosus and implications for percutaneous catheter occlusion. Am J Cardiol 1989; 63: 877-80.

9. Haponiuk I, Chojnicki M, Jaworski R, et al. Delayed closure of multiple muscular ventricular septal defects in an infant after coarctation repair and a hybrid procedure: a case report. Heart Surg Forum 2011; 14: E67-9. 